\title{
Place information in multidigit number comparison
}

\author{
JAMES V. HINRICHS, JANIS L. BERIE, and MOLAAN K. MOSELL \\ University of lowa, lowa City, Iowa 52242
}

\begin{abstract}
How do we use place information in a number comparison task involving multidigit numbers? In four experiments, subjects identified stimulus numbers as larger or smaller than the number 5,000 in a choice reaction time task. As the difference between the number of places in the stimulus number and the number of places in the standard decreased, response time and errors significantly increased. When the number of places was held constant, the type of numeric information extracted depended on the value of the standard $(5,000$ or 5,555$)$. In some cases, irrelevant place information affected choice time.
\end{abstract}

A characteristic feature of the decimal (Arabic) numeration system, the concept of place value, raises some interesting theoretical questions related to the way we process and store numerical information. According to the decimal system, the value or size of a multidigit number is determined by the combined values of its numerals; the value of each numeral is determined by its identity and place (position) within the number. How do we use the dual information of numeral identity and place to determine the relative value of a number? Does the number of places contained within the number alone provide a cue to the number's value? Are the individual numerals processed as single digits or converted to a unitary quantity (magnitude) representation?

Previous investigations have provided some information about the way we process single-digit numbers. Moyer and Landauer (1967) found that the time needed to identify the larger of a pair of single-digit numbers was primarily a function of their difference: the greater the difference, the faster the decision. Since then, the "difference effect" has been reported by several other investigators in a variety of experimental tasks (Aiken \& Williams, 1968; Buckley \& Gillman, 1974; Sekuler, Rubin, \& Armstrong, 1971). Because numerical difference affects the decision time in comparing two numbers in the same way that magnitude difference affects the decision time in comparing two physical stimuli (Welford, 1960), Moyer and Landauer suggested that we convert numbers to analog representations in order to compare them. Banks (1977) has discussed implications of analog representation models and alternative interpretations, especially semantic coding models.

This research was supported by National Institute of Education Grant G-0178 to J. V. Hinrichs. Experiment 1 was conducted as part of an honors thesis at the University of Iowa by the third author under the direction of the first author. We thank Laura Novick for conducting Experiment 4. A preliminary report of the data was presented at the meeting of the Psychonomic Society, Phoenix, Arizona, November 9, 1979. Requests for reprints should be sent to James V. Hinrichs, Department of Psychology, University of Iowa, Iowa City, Iowa 52242.
Hinrichs, Yurko, and Hu (1981) extended the generality of the difference effect to two-digit numbers by examining the use of place information in a task in which two-digit numbers were compared to a standard of 55. The results indicated that both digits were processed as a single value despite the fact that most of the comparisons could have been made by processing the digit in the tens place only. Place information played a role in the comparison process only when the comparison number was in the same decade as the standard.

It appears, at least in some circumstances, that numbers may be cognitively represented as a single value or quantity analogous to a point on a continuum or a physical magnitude. Research supporting this view has involved only one- and two-digit numbers. With numbers of increasing magnitude, more information must be considered; there are more numerals to identify and more places to evaluate. Furthermore, our familiarity with and use of numbers of increasing magnitude decreases. For these reasons, it is interesting to consider the underlying processes involved in comparing large numbers. The purpose of these experiments was to examine the way we process and store place information in a number comparison task involving multidigit numbers.

The most obvious available cue for determining the relative value of a multidigit number is simply the number of places or the length of the number. Certainly row length is a potent determinant of decision time and accuracy for relative quantity judgments for both children and adults. When the number of objects in a row conflicts with the length of the row of objects, children make more judgment errors (Pufall \& Shaw, 1972), and young children may use only the length cue to decide which row has more objects (Gelman, 1972). Although accuracy remains high, adult quantity comparison time is also increased by discrepancies between symbol row length and the number of symbols in the row (Dixon, 1978). Dixon proposed a model of number comparison in which row length is a major factor in determining relative quantity. 
In the number comparison studies reported above, the subjects' task was to select the greater number (quantity) of a set of arbitrary objects or symbols. When the symbols are not arbitrary, and the subjects' task is to determine the relative value of a number, length cues must be integrated with the meaning of the numerals in the number. A central question for the research reported here is the extent to which row length cues (number of places) influence speed and accuracy in understanding number value.

To decide the magnitude of a number, one must assess both the value of individual digits and the number of digits (places) in the number. For example, if the task is to determine if a presented number is greater than or less than 5,000 , the value of the first numeral is insufficient information-the number (but not necessarily the value) of succeeding numerals must also be determined. The number of digits, if not exactly four, is sufficient to make an accurate response, but more information must be extracted if the number of digits in the test number matches the number in the standard.

Note that the simplest process model (evaluate the number of places and then determine the value of the first and succeeding digits) implies that the value of individual digits will not affect decision times unless the number of places in the test number and standard are the same. However, in comparing two-digit numbers, Hinrichs et al. (1981) found that nonsignificant numerals did influence decision times. Furthermore, if response tendencies are associated with each individual numeral in a multidigit number, the tendencies may combine to facilitate or interfere with accurate, rapid decisions. The Stroop interference literature (e.g., Dyer, 1972) demonstrates that even simple, familiar stimuli may evoke competing response tendencies. Within the number comparison task, even decisions between two single digits exhibit strong congruity effects manipulated either by the response label (Banks, Fujii, \& KayraStuart, 1976) or by an interpolated irrelevant numeral (Bennick, 1975). Therefore, in Experiment 1, the effects of the value of the first digit or of the following digits on decision time were also examined.

\section{EXPERIMENT 1}

\footnotetext{
Method

Subjects. Fourteen undergraduates from introductory psychology classes participated in this experiment. The subjects, eight females and six males, were tested in two groups, each group participating in a session lasting approximately $45 \mathrm{~min}$.

Procedure. Each subject sat at a table containing a response panel with a horizontal row of nine buttons. For this experiment, the middle seven buttons were covered with masking tape, and only the buttons on each end were used. Subjects placed the thumb or forefinger of each hand on one of the buttons. One button was labeled "larger" and the other button was labeled "smaller." The side on which the labels were assigned was counterbalanced, with one half of the response panels having "larger" on the left and one half of the panels having "larger" on the right.
}

The subjects were informed that they would be presented with a series of slides containing multidigit numbers. They were instructed to push the button labeled "smaller" when the number was smaller than 5,000 and to push the button labeled "larger" when the number was larger than 5,000 . Subjects were instructed to respond rapidly and to make as few errors as possible.

Dark numerals on a light background, each with an image size of $10 \times 6 \mathrm{~cm}$, were rear-projected onto a vertical screen 1.8 to $3.3 \mathrm{~m}$ from the seated subjects. Depending on the subject's distance from the viewing screen and the number of digits in the display, the visual angle of the presented number ranged from .1 to $1.3 \mathrm{deg}$. A tachistoscopic shutter attached to the projector lens controlled the onset and offset of each slide. Timing of stimulus presentation was under the control of a solid state logic system. The system also recorded errors and RTs to the nearest $.01 \mathrm{sec}$. The stimulus exposure duration (onset to offset) was $1.5 \mathrm{sec}$, and the intertrial interval (offset to onset) was $2 \mathrm{sec}$.

Design and Materials. The number of places within each stimulus numeral and the value of the first digit were the two main independent variables. All possible combinations of number of places (ranging from one to seven places) and first-digit values (ranging from 1 to 9 ) were included, producing 63 unique stimulus configurations. Four exemplars of each place-by-firstdigit combination were generated, and each appeared in one of four different trial blocks. Subjects completed eight blocks of 67 trials each (4 unscored warm-up trials per block were included); the four different trials blocks were each presented twice, producing a total of eight observations per stimulus combination.

The four instances of each of the 63 stimulus combinations were generated digit by digit with a random number table using the following restrictions: (1) The digit 0 was not used. (2) The digit 5 was used only as a first-place number, as a single-digit stimulus number (i.e., a one-place number with the first digit 5), and as the first digit of a multidigit stimulus number, but at no other times. (3) The same digit could appear no more than two times within a single stimulus item. (4) When a digit was repeated within a stimulus number, the two appearances were never adjacent to each other. Four different trial blocks, each containing one instance of the place-by-first-digit combination, were randomly arranged, with the following restrictions: (1) Numbers beginning with the same first digit were not presented consecutively. (2) Numbers containing the same number of places were not presented consecutively. (3) No more than four consecutive stimuli requiring the same response (either "larger" or "smaller") were presented. All numbers were presented without commas.

\section{Results}

Most of the analyses are based on RTs for correct responses only, but some preliminary consideration of errors is necessary. The error rate of individual subjects ranged from $2.8 \%$ to $7.5 \%$ with an overall mean of $4.4 . \%$. Figure 1 presents the error rate for each number of places within the stimulus number; it is clear that only the four-digit numbers posed difficulties. For four-place numbers, the mean error rate was $17.3 \%$, which was significantly different from the $2.2 \%$ error rate for trials excluding four-digit numbers $[F(6,78)=44.02]$. (A significance level of .01 is assumed for all analyses.) The error rate for different first-place digits interacted with number of places $[F(48,624)=1.95]$, but no consistent patterns were discernible.

Also shown in Figure 1 are the mean RTs as a function of the number of places within the stimulus number and the value of the first digit. The sharply declining 


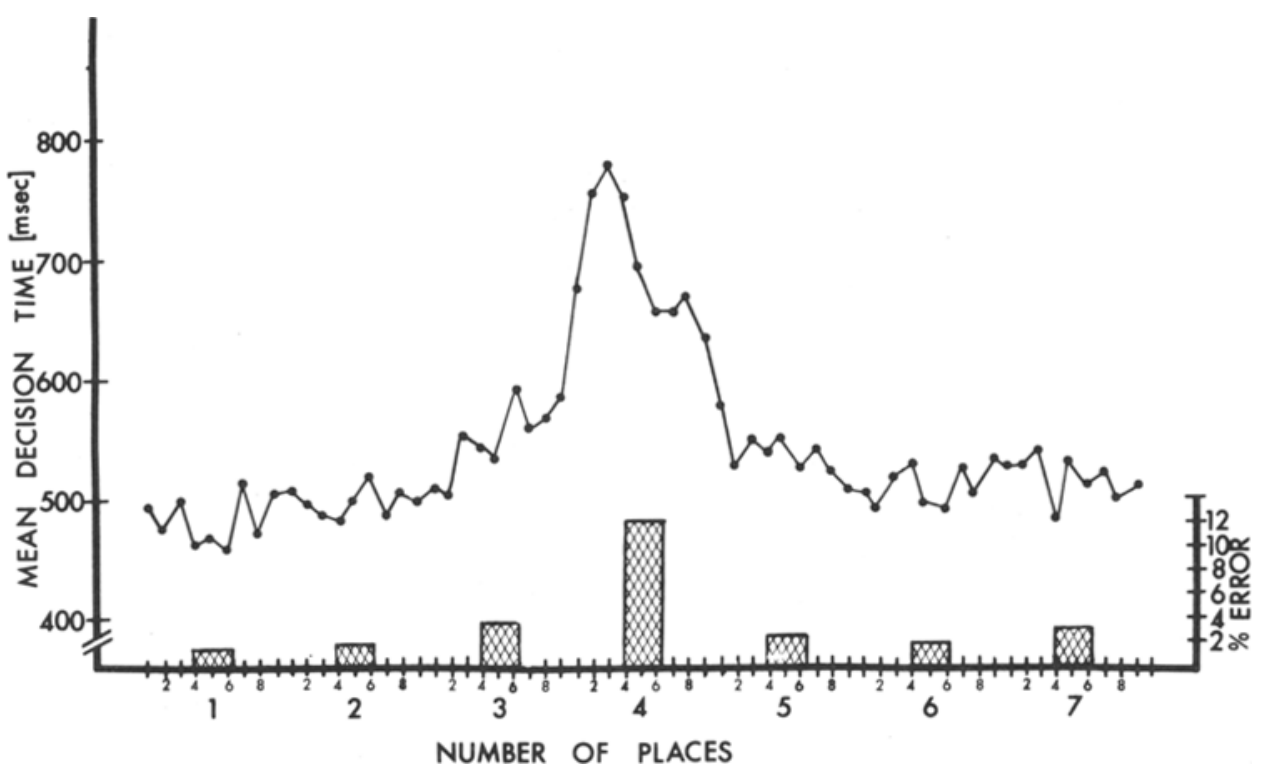

Figure 1. Percent errors and mean RT to decide if a presented number is less than or greater than 5,000 as a function of the number of places and value of the first digit.

decision time when the stimulus number was located farther from the standard reflects the significant effect of number of places $[F(6,78)=35.04]$. As the number of places increased from one to seven, the mean RTs were $483,498,550,696,538,511$, and 516. Although the interaction between number of places and first digit was significant $[F(48,624)=380]$, the main effect of value of the first digit in each number was not $[F(8,104)=$ 1.58 ]. Follow-up analyses indicated reliable first-digit differences with three- and four-place numbers and marginal effects $(p<.05)$ with one-, two-, five-, and six-place numbers. To distinguish systematic effects of the first-place digit, linear trends were tested within each place-number condition. Regression analyses of RT as a function of distance from 5,000, using first digit and number of places as equally spaced units, revealed significant slopes both above $(r=-.70)$ and below $(r=.79)$ the standard. First-digit slopes were reliable for three-digit $(\mathrm{r}=.82)$ and five-digit $(\mathrm{r}=-.73)$ numbers.

Analyses of nonleading digits. The results described above consider only the approximate value of the number as controlled by the number of places and the value of the first digit. Certainly these are the primary determinants of a number's "meaning" or value, but one must also consider the context provided by the digits following the first-place numeral. For example, consider the three five-place numbers 29768,21379 , and 31424 . All are clearly above the 5,000 standard, but there are at least two ways that the nonleading numerals might affect number comparison. The two factors considered are labeled "agreement" and "consistency" cues.

Agreement was coded by the degree of congruency between the value of the first digit in a number and the values of the nonleading digits. Agreement was coded as full, half (first or second half), or none, depending on whether the leading and nonleading numerals were greater than or less than 5 . For example, the number 31424 would be coded as full agreement, 21379 as first half, and 29768 as no agreement. Obviously, single-digit numbers and numbers beginning with 5 could not be classified and were coded into a null category. The assumption to be tested was that the trailing numerals provide a context for deciding whether the presented number is greater than or less than 5,000 . The agreement code indicates the extent to which later numerals are consistent with the first numeral. If there is a tendency to use each digit in generating the correct response, all stimulus numerals may be coded as small or large depending on whether they are greater than or less than 5 ; consequently, the greater the agreement, the shorter the decision time for approximately equal numbers.

An analysis of variance of the three- to seven-place numbers with five levels of agreement yielded no support for an influence of degree of agreement. Although the main effect of place was significant, consistent with the previous analyses $[F(4,52)=29.12]$, there was no agreement effect $[F(4,52)=1.44]$ or interaction $[\mathrm{F}(16,208)=1.21]$. The difference in mean RT over agreement categories was slight: $562,555,565,564$, and $566 \mathrm{msec}$ for the null, zero, first-half, second-half, and full agreement categories, respectively. The two-place numbers permit only null, zero, and full agreement, and obtained mean RTs were 496,496 , and 498 , again showing no difference among the categories. Consequently, there was no evidence that the trailing digits affected the processing of the value of the first digit.

A second possible factor considered was the number of digits consistent with the correct response. For example, fast responses for larger numbers might be a func. tion of the number of individual digits consistent with 
that response, that is, numerals indicating that a "large" response was appropriate. Stimulus numbers were coded as having zero to six consistent response cues, the cue being defined as any digit less than 5 when the number that included it was less than 5,000, and any digit greater than 5 for numbers greater than 5,000 . For example, 312 has three consistency cues and 589 has zero; 31424 has zero cues and 29768 has four. The number of cues ranged from zero to $\mathrm{N}$, where $\mathrm{N}$ is the number of places in the presented number, except that four-digit numbers with zero cues did not exist; the first digit determines the response; hence, at least one digit must be consistent.

Separate analysis of variance for each place value yielded only one marginal effect of consistency cues $[F(5,65)=3.00, p<.02]$ for five-place numbers, but there was no tendency for RTs to decrease as the num. ber of cues increased. All other analyses were nonsignificant.

\section{Discussion}

Both errors and decision times indicate that length of the presented number (i.e., number of places) is a powerful cue in determining whether the number is relatively large (greater than 5,000 ) or relatively small (less than 5,000). Only modest increases in mean RT (approximately $50 \mathrm{msec}$ ) were observed as the difference in number of places between the presented number and the standard decreased from three to one. But when the number of places was the same, mean decision times were slowed by an additional $150 \mathrm{msec}$. Clearly the length of a number affects the time to encode and/or process the meaning of the number. For four-digit numbers, the value of the first numeral must be used to make the correct response. The erratic pattern of response times for four-digit numbers can be attributed to the relatively small number of instances in the range above and below 5,000 and to the high error rate.

Numerals after the first had no apparent effect on decision times. No influence of number context or Stroop-like interference effects in deciding that a number was greater than or less than 5,000 could be detected.

Although both encoding and comparison processes could potentially contribute to the obtained results, attempts were made to minimize the influence of encoding differences. The use of a comparison paradigm in which the standard is held in memory, small visual angles in the presented stimuli, and relatively long exposure durations should tend to reduce encoding contributions to the task. The symmetry of the results for stimuli above and below the standard suggest that differences in encoding large and small numbers played little role in the decision process.

At first glance, the results seem quite consistent with a simple two-stage interpretation of the decision process: (1) Evaluate the number of places and choose smaller if places are less than 4 and choose larger if places are greater than 4. (2) If the number of places is exactly
4, then evaluate the four-digit numbers, with shorter RTs for first digits less than 5 and longer RTs for first digits greater than 5 . However, systematic decreases in RT occurred as the distance from 5,000 increased, even for three- and five-digit numbers, suggesting that the value of the number was affecting the decision time. Obviously, evaluation of length cues and processing of first-digit meaning could proceed in simultaneous or overlapping stages, but the significant influence of the value of the first digit on decision times for three- and five-digit numbers rejects a simple two-stage model of number comparison.

\section{EXPERIMENTS 2 AND 3}

The role of zero in place notation is to serve as a placeholder. The goal of Experiments 2 and 3 was to explore the influence of zeros either before (leading) or after (trailing) the critical numeral. Consider the number 21379 compared to the standard of 5,000 . If the last four places were converted to zeros (20000), we would not expect much decrease in the comparison time (except for the slight reduction in the difference between standard and test stimulus), but would decision times be affected if leading zeros were introduced (e.g., 020000)? As a direct test of the influence of number of places on number comparison, the presence or absence of leading and trailing zeros was manipulated. If number of places per se or, alternatively, the perceptual length of a series of numerals is a critical cue, then holding the number of places constant by filling any empty leading spaces with zeros should reduce the saliency of the place cue and should slow number comparison speed and accuracy.

Introduction of leading or trailing zeros could have different effects, depending on whether the comparison conditions are blocked or mixed. For example, processing 020000 may be different if it occurs only in the context of $000300,080000,000070,006000$, and so forth, than if it occurs in the context of numerals such as $378,80000,70$, and 6487 . Consequently, two separate experiments were conducted: Experiment 2 manipulated the use of zeros as a between-subjects variable, and Experiment 3 used a within-subjects design.

\section{Method}

Subjects. The subjects in Experiment 2 were 48 undergraduates enrolled in an introductory psychology class at the University of lowa, participating in the experiment in order to fulfill part of a class requirement. There were 12 subjects in each of the four stimulus conditions. In Experiment 3, the subjects were 53 different students from the same introductory psychology class. All subjects in Experiments 2 and 3 were tested individually or in pairs.

Design and Materials. As in Experiment 1, the primary variables of interest were the number of places within each number and the value of the first digit. However, in both Experiments 2 and 3 , only two to six-place numbers were included, with values of first digit ranging from 1 to 9 . Thus, 45 unique stimulus combinations were tested. In addition, four stimulus conditions were manipulated in order to assess the role of place cues. The four conditions formed a 2 by 2 factorial com- 
bination of presence or absence of leading and trailing zeros. For the leading zeros condition, the zeros replaced all numerals after the first digit. Therefore, the four experimental conditions were (1) the no-zeros condition, (2) the leading zeros condition, (3) the trailing zeros condition, and (4) the leading-trailing zeros condition. The no-zeros condition was identical to the condition used in Experiment 1 (e.g., 367). Each of the numbers in the leading zeros condition was preceded by a series of leading zeros so that exactly six digits were always presented. The number of leading zeros added was dependent on the number of significant digits in the multidigit number (e.g., the number 367 in the no-zeros condition was presented as 000367 in the leading zeros condition). No leading zeros were added to six-digit numbers. In the trailing zeros condition, each stimulus number was presented with only one significant digit followed by a series of zeros (e.g., the number 367 in the no-zeros condition was presented as 300 in the trailing zeros condition). Characteristics of both Conditions 2 and 3 were included in the leading-trailing zeros condition (e.g., the number 367 in the no-zeros conditon was presented as 000300 in the leadingtrailing zeros condition).

In Experiment 2, the experimental condition (no zeros, leading zeros, trailing zeros, or leading-trailing zeros) served as a between-subjects variable. In Experiment 3 , the experimental condition was a within-subjects variable; subjects received all four conditions presented in a random order.

The same stimulus numbers used in Experiment 1 were used as the no-zeros condition in Experiments 2 and 3; however, only two- through six-place numbers were included. Stimulus items corresponding to those used in the no-zeros condition were reconstructed according to the requirements of the remaining three experimental conditions. Note that because no more than six digits were ever presented, stimulus items in the sixdigit leading zeros condition were identical to the items in the no-zeros condition and could not be distinguished in mixed trial blocks (Experiment 3).

In Experiment 2, four instances of each of the 45 (nine first digits by five places) stimulus combinations for each experimental condition were included. Each instance appeared in one of four different trial blocks per condition (with four warm-up trials per block) that were repeated twice. Thus, eight observations for each place-by-first-digit combination for each stimulus condition were recorded.

In Experiment 3, each subject received all four stimulus conditions. Only two replications of each of the 180 stimulus combinations (nine first digit by five place by four conditions) were included. Eight different trial blocks of 45 test trials plus 4 warm-up trials were constructed. In each trial block, an approximately equal number of instances of each stimulus condition were presented, and each of the 45 place-by-first-digit combinations was represented by one of the four stimulus conditions. In both experiments, order of the eight trial blocks was randomized for each set of subjects.

Procedure. As in Experiment 1, subjects were instructed to decide if the multidigit number presented on each trial was larger than or smaller than the standard of 5,000. The target numbers appeared as $7 \times 4 \mathrm{~cm}$ numerals on a screen approximately $1.5 \mathrm{~m}$ from the seated subjects. The subjects held a response panel with two response keys arranged side by side and indicated their choice on each trial by pressing one of the two response keys labeled either "larger" or "smaller." The assignment of response button labels was counterbalanced in each experiment, with one half the subjects having "larger" on the right side and one half having "larger" on the left side. The experimental procedure was automated and under the control of a PDP-8 minicomputer with the same timing as in the first experiment.

\section{Results}

Because error rates varied considerably across subjects and conditions, both errors and RTs for correct responses were analyzed. A significance level of .01 was again adopted throughout, but the high degree of statistical power resulted in many higher order interactions. Only those interactions relevant to the hypothesis under investigation or exhibiting consistent trends across conditions and experiments are reported.

Experiment 2. The overall error rates were generally low $(.5 \%$ to $4.5 \%)$ in all conditions except for the fourplace numbers immediately adjacent to the standard, for which the rates ranged from $15.4 \%$ to $23.0 \%$. Obviously, the effect of number of places on errors was significant $[F(4,176)=181.67]$, as was the interaction with stimulus condition $[F(12,176)=2.91]$. The Condition by Number of Places interaction was primarily attributable to more errors in the four-place numbers with leading zeros $[F(4,176)=6.95]$. Most important, neither the presence of leading or trailing zeros nor their combination affected the overall error rate $(F<1)$. Thus, although the number of significant places in the test numbers greatly influenced the error rate, the presence of superfluous or placeholding zeros did not.

The pattern of results for RTs was quite different. Although substituting trailing zeros for "nonsignificant" digits did not affect the mean RT $(F<1)$, the introduction of extra leading zeros did significantly increase mean decision time (from 548 to $630 \mathrm{msec}$ ) $[\mathrm{F}(1,44)=$ $8.48]$. The interaction of leading and trailing zeros was not significant $[F(1,44)=2.98]$. The main effect of number of places was also significant $[F(4,176)=$ $196.14]$, as was the interaction with conditions $[F(4,176)=3.78]$. However, the order of results was the same at each place value: The "round" numbers (trailing zeros condition) produced the shortest decision times (mean RT over all places was $519 \mathrm{msec}$ ), followed by the no-zeros condition ( $577 \mathrm{msec}$ ), and the leading zeros condition $(611 \mathrm{msec})$. The top panel of Figure 2 presents the mean RT for each presentation condition and number of places.

The leading zeros condition was the only condition to exhibit consistent effects of the value of the first-place digit within each number. Regression analyses within each number of places revealed significant slopes for two-, three-, and five-place numbers for the leading zeros condition, but only for five-place numbers in the no-zeros and trailing zeros conditions. (Slopes were highest for all conditions in the four-place numbers, as expected, but dividing the functions into two segments, one above the 5,000 target and one below, reduced the number of observations to the point that reliable differences were not obtained.) Reliable effects of the value of the first significant digit across several place values in the leading zeros condition suggest that first-digit value most affected number comparison when place cues were reduced by the presence of extra leading zeros. In other conditions, other place cues were apparently available and sufficient to determine the relative value of the number without processing the value of the first significant digit.

Experiment 3. A very similar pattern of results 


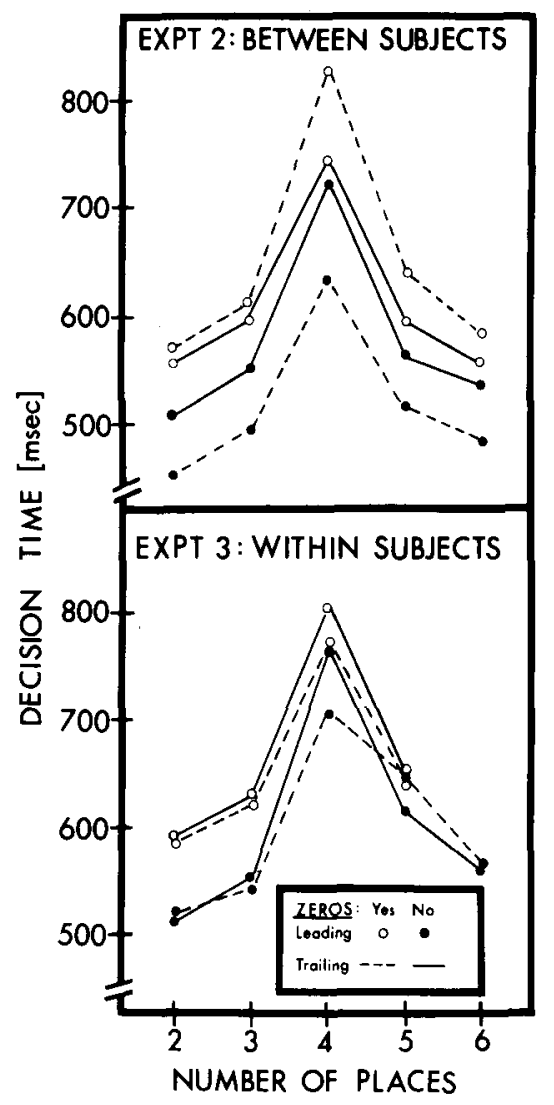

Figure 2. Mean RT as a function of number of places in the presented number and presence of zeros before and after the most significant digit.

emerged when the stimulus conditions were mixed (Experiment 3) rather than tested separately (Experiment 2). The overall error rate was $4.3 \%$, and, as in Experiment 2, the number of places was the primary factor affecting errors. Presentation of four-place numbers produced $11.4 \%$ errors, and all other conditions combined yielded only $2.5 \%$ errors. The presence of neither leading zeros nor trailing zeros affected the error rate $(F<1$ in both cases), although the interaction did approach significance $[F(1,54)=6.79$, $.01<p<.02]$. Leading and trailing zeros did not affect the distribution of errors as a function of the number of places, although several significant interactions of conditions with the value of the first digit were noted.

The high statistical reliability generated by testing a relatively large number of subjects and using RT as the dependent variable resulted in almost all factors and interactions producing significant differences. Number of places, presence of leading and trailing zeros, and all interactions were significant, with the noteworthy exception that the interaction between leading and trailing zeros was not significant (cf. Figure 2, lower panel).

The mean RTs for the stimulus conditions were as follows: The trailing zeros condition had the shortest mean RT ( $603 \mathrm{msec}$ ), followed by the no-zeros condition (614 $\mathrm{msec})$, and the leading-trailing zeros condition $(657 \mathrm{msec})$. The leading zeros condition had the longest mean RT (673 msec). "Rounding," that is, replacing all digits after the first with zeros, reduced overall decision time by approximately $15 \mathrm{msec}$, but the effect varied, with number of places $[\mathrm{F}(3,156)=21.87]$ being greatest $(48 \mathrm{msec})$ in the four-place numbers and even reversing slightly $(4 \mathrm{msec})$ for five- and six-place numbers. Introducing extra leading zeros consistently reduced decision time by approximately $60 \mathrm{msec}$ across all levels of number of places.

Analyses of the effect of first digit within each place condition yielded generally steeper slopes than reported for Experiment 2, but the correlations with comparison time were smaller, obtaining significance only in the five-place number conditions. The pattern of results suggests that with mixed presentation of stimulus conditions, subjects used a variety of cues in order to determine whether the presented number was greater than or less than 5,000 and relied on the value of the leading significant digit only when the numbers were four- or five-place numbers.

\section{Discussion}

The combined results of Experiments 2 and 3 indicate that number of places, that is, the number of digits presented, is the most potent cue for discriminating the relative value of multidigit numbers. Several observations support this interpretation: (1) As in Experiment 1 , decision times were markedly increased when the comparison numbers had the same number of digits as the standard $(5,000)$. (2) The introduction of superfluous leading digits, which reduced the effectiveness of number of digits as a discrimination cue, consistently increased decision time, regardless of whether all the presented numbers were embedded in extra zeros (Experiment 2) or mixed with other types of numbers (Experiment 3). (3) Another potential factor in number comparison, "rounding," had only a small effect on decision time, primarily when the rounding cue was isolated and other types of numbers were not encountered (Experiment 2). Curiously, rounding appears to facilitate number comparison not by emphasizing the value of the leading nonzero numeral, but rather by providing a more salient cue for determining the number of places in the presented number.

\section{EXPERIMENT 4}

The previous experiments were primarily concerned with the effectiveness of number of places as a cue in number comparison. Experiment 4 examined the role of place value when number of places did not vary. The results of the first three experiments clearly demonstrate that when number of places is an available cue, the value of the first-place digit affects RT only when the number of places is close to the standard value. The central question of the last experiment was what numerical information is extracted in the attempt to decide whether the presented number is larger or smaller than the standard when number length is held constant. 
There are several closely related issues. Clearly, when all numbers have the same number of places, the value of the numerals in each significant place must be compared. Are the numerals evaluated only to the place necessary to make the decision, or is additional information gained (from the nonsignificant digits)? In other words, are numerals processed in a strict left-toright order, terminating at the location with the critical information, or are all digits considered and used in the comparison? In two-digit number comparisons, Hinrichs et al. (1981) found evidence that ones-place digits affected RT even when only tens-place digits were needed to make the comparison. Use of four-digit numbers permits closer examination of the influence of nonsignificant digits.

What effect does the precision of the comparison standard have on the decision process? Requiring subjects to decide if a number is above or below 5,555 demands evaluation of more places than a standard of 5,000 if numbers close to the standard are presented. The proportion of test values close to the standard may also affect the comparison process. Experiment 4 examined the comparison process in four-digit numbers, varying the value of the standard and the distribution of test numbers.

\section{Method}

Subjects. The subjects were 34 University of Iowa undergraduates who participated to partially fulfill the requirements of an introductory psychology course. The data from two subjects were excluded from the analyses due to equipment problems. Data analyses were performed on data from 22 females and 10 males. The ratio of females to males in each experimental group was approximately the same.

Procedure. The basic procedures and equipment were the same as in Experiment 1. Subjects were told that they would be presented with a series of slides containing four-digit numbers. They were to decide as quickly and accurately as they could whether each number was larger or smaller than the standard number (either 5,000 or 5,555 , depending on the group), which they were instructed to hold in memory. Each subject saw five trays of slides. The first of these contained 24 practice slides. The remaining four trays contained 130 slides, the first 10 of which were unscored warm-up trials.

Design and Materials. The two main variables of interest were the value of the standard $(5,000$ or 5,555$)$ and the distribution of the test numbers around the standard (geometric or arithmetic). The stimuli were all four-digit numbers within a distance of 4,000 from the standard: $1,000-9,000$ for the standard of 5,000 and $1,555-9,555$ for the standard of 5,555 . For each distribution and standard, 120 test slides were constructed. Each set of 120 slides was presented in a separate slide tray, and within each tray, the slides were randomly arranged in blocks of 40 according to the following constraints: (1) There were an equal number of slides above and below the standard. (2) No more than four consecutive stimuli required the same response (either "larger" or "smaller"). (3) Each block was a microcosm of the parent distribution.

The value of the standard was a between-subjects variable in order to avoid confusing subjects by requiring them to change reference points in midsession. Distribution, however, was a within-subjects variable. Half the subjects saw the geometrically distributed numbers first and the arithmetically distributed numbers second, and the other half saw the slides in reverse order. Each slide tray was presented twice. In addition, two different randomizations were constructed for each slide tray, with Sequence A for the geometric distribution yoked to Sequence $A$ for the arithmetic distribution for presentation purposes, and so forth. This 2 (standard) by 2 (slide tray/ distribution order) by 2 (slide sequence within a tray) design resulted in the testing of eight groups of subjects.

For the geometric distribution, there were equal numbers of stimuli within each power of 10 around the standard. Of the 120 test slides/distribution, there were 36 numbers in the intervals $\pm 10, \pm 100$, and $\pm 1,000$ of the standard and 12 numbers in the interval $\pm 4,000$ of the standard (based on 36 numbers within 10,000 of the standard). For the arithmetic distribution, there were three stimuli within each successive \pm 100 interval around the standard. For both of the distributions, each interval around the standard excluded the previous intervals. Thus, for the geometric distribution, the interval $\pm 1,000$ included numbers at a distance greater than 100 but less than 1,000 from the standard.

The above constraints meant that a maximum of 42 stimuli could overlap between the two distributions ( 3 stimuli/100 up to $\pm 1,000$ plus 4 stimuli/ 1,000 thereafter). These common numbers were chosen first, conforming to the constraints of both distributions, and the remaining numbers for each distribution were filled in as needed. All stimuli were chosen by first using a random number table to select distances from the standard according to the appropriate distributional constraints. Thus, although the actual stimuli for each standard were different, the distributions of the distances of the stimuli from their respective standards were identical.

The 24 practice slides were selected based on a mixed distribution combining the arithmetic and geometric distributions used for the test stimuli. There were 8 numbers within 100 of the standard, 6 numbers within 1,000 , and 10 numbers within 4,000 . The practice slides for the two standards were based on the same set of 24 distances. The 10 warm-up slides for the test trays were also based on this intermediate distribution.

\section{Results}

The overall error rate was $2.2 \%$. Error rates were between $1.6 \%$ and $1.8 \%$ for the 5,000 geometric, 5,000 arithmetic, and 5,555 arithmetic distributions, but $3.6 \%$ of responses were errors for the 5,555 geometric distribution.

Preliminary analyses revealed no consistent practice effects across presentation orders; all further analyses are based on results collapsed over order. Although the type of stimulus distribution, arithmetic or geometric, greatly affects the number of observations available at various points in the RT functions, it had little effect on mean RT at comparable values. As anticipated, the value of the standard greatly influenced the RT functions.

For the standard of 5,000 , the effect of the value of the first-place differed for the two distributions. Regression analysis was used to identify systematic first-place digit effects on observed mean RT. For the arithmetic distribution, as the absolute value of the difference between the presented first-digit and the standard value (5) increased, mean RT decreased. The slope of the best fitting regression line $(-22.8)$ was significantly different from zero $[t(1,6)=5.94, p<.01]$ and symmetrical (i.e., the slopes of the segments above and below the standard did not differ, $t=1.13$ ). However, 
compared to the differences reported below for the standard of 5,555, the magnitude of the distance effect was relatively small. Mean RTs ranged from 671 for the most distant first-place digits $(1,8)$ to 740 for the closest $(4,5)$. For the geometric distribution, no distance effect was observed. The best fitting regression function was essentially flat (slope $=4.6 ; \mathrm{t}<1$ ).

For the 5,555 standard and for both distributions, the value of the first digit strongly influenced the observed decision times. The observed slopes were nearly identical, -44.2 for the arithmetic distribution and -40.5 for the geometric ( $p<.01$ in both cases). Mean RTs ranged from 706 at the most distant first-place digit $(1,9)$ to 946 at the closest $(5)$. Although small numbers of observations and missing data preclude statistical analyses, later significant digits produced a similar pattern of longer RTs as the value of the significant digit approached 5. For example, RTs to stimuli in the range 5,000-5,999 varied from approximately $850 \mathrm{msec}$ for stimuli of $5,000-5,100$ and $5,900-5,999$ to $1,000 \mathrm{msec}$ for stimuli of $5,500-5,599$. Even stimuli \pm 10 from the standard of 5,555 produced mean RTs ranging from 974 for $5,555 \pm 4$ to 1073 for $5,555 \pm 1$.

Nonsignificant digits also influences decision times when the standard was 5,555 . For example, consider numbers in the range 4,000-4,999 and 6,000-6,999. Obviously, the value of the first-place digit is sufficient to decide whether the presented number is larger or smaller than the standard. However, as the distance from the standard increased, the mean RT decreased from $785 \mathrm{msec}$ for stimuli in the $4,900-4,999$ and 6,000-6,099 intervals to $735 \mathrm{msec}$ for stimuli from 4,000-4,099 and 6,900-6,999. No distance effects were evident when the standard was 5,000 .

The RT data for 5,555 standard conditions were well described by functions based on the difference between the presented number and the standard: $\mathrm{RT}=-172 \log \mathrm{D}+1294$ for the arithmetic distribution, and $\mathrm{RT}=-111 \log \mathrm{D}+1113$ for the geometric distribution, where $\log D$ is the logarithm of the absolute difference between the test stimulus and the standard. The observed correlations were -.88 and -.87 , respectively.

\section{Discussion}

The results clearly demonstrate that when number of places was held constant, the subjects processed individual digit information according to the demands of the task. When the demarcation between larger and smaller responses was fairly sharp, as in the case of the 5,000 standard with a geometric distribution of stimuli, subjects essentially performed a simple classification task. The geometric distribution places most stimuli in the 4,000-5,999 range, allowing subjects to identify just the leading digit and respond appropriately. By both subjects' self-reports and RT data, the larger-smaller decision was reduced to a binary classification. The occasional number beginning with a digit other than
4 or 5 was easily classified with only a small increase in RT. With the same standard but with a rectangular (arithmetic) distribution of stimuli, subjects responded to the leading digit much as they would to a singledigit number compared to 5 (Sekuler et al., 1971). Mean RT decreased as the absolute difference between the first-place digit and 5 increased, but second-place and later digits had no effect on RT.

A comparison standard of 5,555 produced a more thorough evaluation of the value of each four-digit test number. For both distributions, not surprisingly, RTs increased sharply as the test stimuli approached 5,555, requiring the identification of hundreds-, tens-, and onesplace digits before a decision could be made. More surprising, however, was the observation that nonsignificant digits also affected decision time. Compared to the magnitude of the effect of significant digits, the influence of nonsignificant digits is small (on the order of $50 \mathrm{msec}$ compared to $150 \mathrm{msec}$ for digits in the hundreds position) but would appear to reject a serial selfterminating model of place-by-place digit processing.

\section{GENERAL DISCUSSION}

Multidigit number comparison appears to require several processes, probably overlapping in their execution. The first process and the most importnat comparison cue is merely the identification of the number of places in the presented number. If the place cue is not camouflaged, subjects apparently make a relatively rapid psychophysical judgment of the number of places (or length of the number) to determine if it is greater than the four-place standard number held in memory. Embedding the test number in the context of superfluous leading zeros significantly increased the time required for this process without appearing to influence other components of the comparison.

If the test number contained the same number of places as the standard (four) or was relatively difficult to discriminate from a four-place number (i.e., threeand five-place numbers), the value of the leading nonzero numeral exerted an influence on comparison time, demonstrating a second process in multidigit number comparison. Three observations support the assumption of this second component: First, increasingly steeper slopes of RT functions occurred as the value of the number approached the value of the standard $(5,000)$. Second, replacing numerals after the first with zeros ("rounding") had different effects depending on other contextual cues. Outside the range in which the first digit was an effective variable, rounding appeared to have no effect (cf. Figure 2). For numbers close to the standard, rounding effects were more complicated; if leading zeros were also present, comparison time increased, suggesting that the place discrimination process was impaired. But if only the nonsignificant digits were rounded to zero, comparison time was 
slightly reduced. [However, it should be noted that the reduction in RT could be attributed either to rounding per se (i.e., increased salience of the leading digit) or to the change in the mean difference between the standard and the presented number, increasing the difference for numbers less than 5,000 and decreasing the difference for numbers greater than 5,000. The observed results were ambiguous; in Experiment 2, rounding reduced mean RTs for four-place numbers below 5,000 and slightly increased RTs above 5,000 compared to the no-zeros condition. In Experiment 3, rounding reduced RT both above and below 5,000.]

The third observation supporting a digit evaluation process was provided by Experiment 4: When precise number comparisons are required (e.g., employing a standard of 5,555), individual digits affect decision time even when they are not required to decide if the test stimulus is larger or smaller than the standard. The results are similar to those obtained by Hinrichs et al. (1981) with two-digit number comparisons.

All of these results are descriptively consistent with the observation that decision time increases as the log. arithm of the absolute difference between presented value and standard increases. The processes generating this function remain uncertain, however. Subjects could either be converting numerical information to some magnitude (analog) representation and making direct psychophysical comparisons or combining physical length cues (number of places) with symbolic evaluation (digit value) to determine relative magnitude of multidigit numbers.

\section{REFERENCES}

Aiken, L. R., \& WiLliams, E. N. Three variables related to reac- tion time to compare single-digit numbers. Perceptual and Motor Skills, 1968, 27, 199-206.

BANKs, W. P. Encoding and processing of symbolic information in comparative judgments. In G. H. Bower (Ed.), The psychology of learning and motivation (Vol. 11). New York: Academic Press, 1977.

Banks, W. P., Fujil, M., \& Kayra-Stuart, F. Semantic congruity effects in comparative judgments of magnitudes of digits. Journal of Experimental Psychology: Human Perception and Performance, 1976, 2, 435-447.

BENNick, C. The effects of stimulus context on the time required for choosing the larger or the smaller of two digits. Bulletin of the Psychonomic Society, 1975, 5, 18-20.

Buckley, P. B., \& Gillman, C. B. Comparisons of digits and dot patterns. Journal of Experimental Psychology, 1974, 103, 1131-1136.

Dixon, P. Numerical comparison processes. Memory \& Cognition, 1978, 6, 454-461.

DYer, F. N. The Stroop phenomenon and its use in the study of perceptual, cognitive, and response processes. Memory \& Cognition, 1973, 1, 106-120.

Gelman, R. The nature and development of early number concepts. In H. W. Reese (Ed.), Advances in child development and behavior (Vol. 7). New York: Academic Press, 1972.

Hinrichs, J. V., Yurko, D. S., \& Hu, J.-M. Two-digit number comparison: Use of place information. Journal of Experimental Psychology: Human Perception and Performance, 1981, 7, 890 901.

Moyer, R. S., \& Landauer, T. K. Time required for judgments of numerical inequality. Nature, 1967, 215, 1519-1520.

Pufall, P. B., \& Shaw, R. E. Precocious thought on number: The long and short of it. Developmental Psychology, 1972, 7, 62-69.

Sexuler, R., Rubin, E., \& Armstrona, R. Processing numerical information: A choice time analysis. Journal of Experimental Psychology, 1971, 90, 75-80.

WELFORD, A. T. The measure of sensory-motor performance: Survey and reappraisal of twelve years' progress. Ergonomics, $1960,3,183-230$.

(Received for publication January 11, 1982; revision accepted May 23, 1982.) 